\title{
THEORY OF STOCHASTIC DIFFERENTIAL EQUATIONS WITH JUMPS AND APPLICATIONS
}

MATHEMATICAL AND ANALYTICAL TECHNIQUES WITH APPLICATIONS TO ENGINEERING 
MATHEMATICAL AND ANALYTICAL TECHNIQUES WITH APPLICATIONS TO ENGINEERING

Alan Jeffrey, Consulting Editor

Published:

Inverse Problems

A. G. Ramm

Singular Perturbation Theory

R. S. Johnson

Methods for Constructing Exact Solutions of Partial Differential Equations with Applications

S. V. Meleshko

Stochastic Differential Equations with Applications

R. Situ

Forthcoming:

The Fast Solution of Boundary Integral Equations

S. Rjasanow and O. Steinbach 
THEORY OF STOCHASTIC DIFFERENTIAL EQUATIONS WITH JUMPS AND APPLICATIONS

MATHEMATICAL AND ANALYTICAL TECHNIQUES WITH APPLICATIONS TO ENGINEERING

RONG SITU

囪 Springer 
Library of Congress Cataloging-in-Publication Data

Theory of Stochastic Differential Equations with Jumps and Applications: Mathematical and Analytical Techniques with Applications to Engineering By Rong Situ

\section{ISBN-10: $0-387-25083-2 \quad$ e-ISBN 10: $0-387-25175-8$}

Printed on acid-free paper.

ISBN-13: 978-0387-25083-0 e-ISBN-13: 978-0387-25175-2

(c) 2005 Springer Science+Business Media, Inc.

All rights reserved. This work may not be translated or copied in whole or in part without the written permission of the publisher (Springer Science+Business Media, Inc., 233 Spring Street, New York, NY 10013, USA), except for brief excerpts in connection with reviews or scholarly analysis. $\mathrm{U}$ se in connection $w$ ith a ny form of in formation s torage a nd r etrieval, electronic adaptation, computer software, or by similar or dissimilar methodology now know or hereafter developed is forbidden.

The use in this publication of trade names, trademarks, service marks and similar terms, even if the are not identified as such, is not to be taken as an expression of opinion as to whether or not they are subject to proprietary rights.

Printed in the United States of America. (BS/DH)

$\begin{array}{lllllllllll}9 & 8 & 7 & 6 & 5 & 4 & 3 & 2 & \end{array} \quad$ SPIN 11399278

springeronline.com 


\section{Contents}

Preface $\quad$ xi

Acknowledgement $\quad$ xvii

Abbreviations and Some Explanations xix

I Stochastic Differential Equations with Jumps in $R^{d}$

1 Martingale Theory and the Stochastic Integral for Point Processes

1.1 Concept of a Martingale. . . . . . . . . . . . . . 3

1.2 Stopping Times. Predictable Process . . . . . . . . . . 5

1.3 Martingales with Discrete Time . . . . . . . . . . . 8

1.4 Uniform Integrability and Martingales . . . . . . . . . . 12

1.5 Martingales with Continuous Time . . . . . . . . . . 17

1.6 Doob-Meyer Decomposition Theorem . . . . . . . . . . . . 19

1.7 Poisson Random Measure and Its Existence . . . . . . . . . 26

1.8 Poisson Point Process and Its Existence . . . . . . . . . 28

1.9 Stochastic Integral for Point Process. Square Integrable Mar-

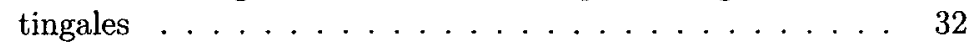

2 Brownian Motion, Stochastic Integral and Ito's Formula 39 
2.1 Brownian Motion and Its Nowhere Differentiability . . . . . 39

2.2 Spaces $\mathcal{L}^{0}$ and $\mathcal{L}^{2} \ldots \ldots \ldots \ldots \ldots . \ldots . \ldots . \ldots . \ldots$

2.3 Ito's Integrals on $\mathcal{L}^{2} \ldots \ldots \ldots \ldots$. . . . . . . . . 44

2.4 Ito's Integrals on $\mathcal{L}^{2, l o c} \ldots \ldots \ldots \ldots \ldots$

2.5 Stochastic Integrals with respect to Martingales . . . . . . . 49

2.6 Ito's Formula for Continuous Semi-Martingales . . . . . . . 54

2.7 Ito's Formula for Semi-Martingales with Jumps . . . . . . . 58

2.8 Ito's Formula for d-dimensional Semi-Martingales. Integration by Parts. . . . . . . . . . . . . . . . . 62

2.9 Independence of BM and Poisson Point Processes . . . . . . 64

2.10 Some Examples . . . . . . . . . . . . . . . . 65

2.11 Strong Markov Property of BM and Poisson Point Processes 67

2.12 Martingale Representation Theorem . . . . . . . . . 68

3 Stochastic Differential Equations $\quad \mathbf{7 5}$

3.1 Strong Solutions to SDE with Jumps . . . . . . . . . . 75

3.1 .1 Notation. . . . . . . . . . . . . 75

3.1.2 A Priori Estimate and Uniqueness of Solutions . . . 76

3.1.3 Existence of Solutions for the Lipschitzian Case . . . 79

3.2 Exponential Solutions to Linear SDE with Jumps . . . . . . 84

3.3 Girsanov Transformation and

Weak Solutions of SDE with Jumps . . . . . . . 86

3.4 Examples of Weak Solutions . . . . . . . . . . . . . 99

4 Some Useful Tools in Stochastic Differential Equations 103

4.1 Yamada-Watanabe Type Theorem . . . . . . . . . . . . 103

4.2 Tanaka Type Formula and Some Applications . . . . . . . . 109

4.2.1 Localization Technique . . . . . . . . . . . . . 109

4.2.2 Tanaka Type Formula in $d$-Dimensional Space . . . 110

4.2.3 Applications to Pathwise Uniqueness and Convergence of Solutions . . . . . . . . . . . . . 112

4.2.4 Tanaka Type Formual in 1-Dimensional Space . . . 116

4.2.5 Tanaka Type Formula in The Component Form . . . 121

4.2.6 Pathwise Uniqueness of solutions . . . . . . . . . 122

4.3 Local Time and Occupation Density Formula . . . . . . . . 124

4.4 Krylov Estimation . . . . . . . . . . . . . . . . . . 129

4.4.1 The case for 1-dimensional space . . . . . . . 129

4.4.2 The Case for $d$-dimensional space . . . . . . . . 130

4.4.3 Applications to Convergence of Solutions to SDE with Jumps . . . . . . . . . . . . . . 133

5 Stochastic Differential Equations with Non-Lipschitzian Coefficients

5.1 Strong Solutions. Continuous Coefficients with $\rho$-Conditions 140

5.2 The Skorohod Weak Convergence Technique . . . . . . . 145 
5.3 Weak Solutions. Continuous Coefficients . . . . . . . . . 147

5.4 Existence of Strong Solutions and Applications to ODE . . 153

5.5 Weak Solutions. Measurable Coefficient Case . . . . . . . 153

\section{Applications}

6 How to Use the Stochastic Calculus to Solve SDE

6.1 The Foundation of Applications: Ito's Formula and Girsanov's

Theorem . . . . . . . . . . . . . . . 163

6.2 More Useful Examples . . . . . . . . . . . . . . 167

7 Linear and Non-linear Filtering

7.1 Solutions of SDE with Functional Coefficients and Girsanov

Theorems .................... 169

7.2 Martingale Representation Theorems (Functional Coefficient Case) . . . . . . . . . . . . . . . . 177

7.3 Non-linear Filtering Equation . . . . . . . . . . . . . . . . 180

7.4 Optimal Linear Filtering . . . . . . . . . . . . . . . . . . 191

7.5 Continuous Linear Filtering. Kalman-Bucy Equation . . . . 194

7.6 Kalman-Bucy Equation in Multi-Dimensional Case . . . . . 196

7.7 More General Continuous Linear Filtering . . . . . . . . . . 197

7.8 Zakai Equation . . . . . . . . . . . . . . . . 201

7.9 Examples on Linear Filtering . . . . . . . . . . . . 203

8 Option Pricing in a Financial Market and BSDE 205

8.1 Introduction . . . . . . . . . . . . . . . . 205

8.2 A More Detailed Derivation of the BSDE for Option Pricing 208

8.3 Existence of Solutions with Bounded Stopping Times . . . . 209

8.3.1 The General Model and its Explanation . . . . . . 209

8.3.2 A Priori Estimate and Uniqueness of a Solution . . . 213

8.3.3 Existence of Solutions for the Lipschitzian Case . . . 215

8.4 Explanation of the Solution of BSDE to Option Pricing . . 219

8.4.1 Continuous Case . . . . . . . . . . . . . . 219

8.4 .2 Discontinuous Case . . . . . . . . . . 220

8.5 Black-Scholes Formula for Option Pricing. Two Approaches 223

8.6 Black-Scholes Formula for Markets with Jumps . . . . . . . 229

8.7 More General Wealth Processes and BSDEs . . . . . . . . . 234

8.8 Existence of Solutions for Non-Lipschitzian Case . . . . . 236

8.9 Convergence of Solutions . . . . . . . . . . . . . 239

8.10 Explanation of Solutions of BSDEs to Financial Markets . . 241

8.11 Comparison Theorem for BSDE with Jumps. . . . . . . . . 243

8.12 Explanation of Comparison Theorem. Arbitrage-Free Market 250

8.13 Solutions for Unbounded (Terminal) Stopping Times . . . . 254

8.14 Minimal Solution for BSDE with Discontinuous Drift . . . . 258 
8.15 Existence of Non-Lipschitzian Optimal Control. BSDE Case 262

8.16 Existence of Discontinuous Optimal Control. BSDEs in $\mathrm{R}^{1}$. 267

8.17 Application to PDE. Feynman-Kac Formula . . . . . . . . 271

9 Optimal Consumption by H-J-B Equation and Lagrange $\begin{array}{ll}\text { Method } & 277\end{array}$

9.1 Optimal Consumption . . . . . . . . . . . . . 277

9.2 Optimization for a Financial Market with Jumps by the Lagrange Method . . . . . . . . . . . . 279

9.2.1 Introduction . . . . . . . . . . . 280

9.2 .2 Models . . . . . . . . . . . . . . . 280

9.2.3 Main Theorem and Proof . . . . . . . . . . . 282

9.2.4 Applications ................. . . 286

9.2.5 Concluding Remarks . . . . . . . . . . . . 290

10 Comparison Theorem and Stochastic Pathwise Control 291

10.1 Comparison for Solutions of Stochastic Differential Equations 292

10.1.1 1-Dimensional Space Case . . . . . . . . . . . 292

10.1.2 Component Comparison in $d$-Dimensional Space . . 293

10.1.3 Applications to Existence of Strong Solutions. Weaker Conditions. . . . . . . . . . . . . . 294

10.2 Weak and Pathwise Uniqueness for 1-Dimensional SDE with Jumps . . . . . . . . . . . . . . . . . . . 298

10.3 Strong Solutions for 1-Dimensional SDE with Jumps . . . . 300

10.3.1 Non-Degenerate Case . . . . . . . . . . . . 300

10.3.2 Degenerate and Partially-Degenerate Case . . . . . 303

10.4 Stochastic Pathwise Bang-Bang Control for a Non-linear System . . . . . . . . . . . . . . . 312

10.4.1 Non-Degenerate Case . . . . . . . . . . . 312

10.4.2 Partially-Degenerate Case . . . . . . . . . . 316

10.5 Bang-Bang Control for $d$-Dimensional Non-linear Systems 319

10.5.1 Non-Degenerate Case . . . . . . . . . . . . 319

10.5.2 Partially-Degenerate Case . . . . . . . . 322

11 Stochastic Population Control and Reflecting SDE 329

11.1 Introduction . . . . . . . . . . . . . . . 330

11.2 Notation . . . . . . . . . . . . . . . . . 332

11.3 Skorohod's Problem and its Solutions . . . . . . . . 335

11.4 Moment Estimates and Uniqueness of Solutions to RSDE . 342

11.5 Solutions for RSDE with Jumps and with Continuous Coefficients . . . . . . . . . . . . . . 345

11.6 Solutions for RSDE with Jumps and with Discontinuous Coefficients . . . . . . . . . . . . . . 349

11.7 Solutions to Population SDE and Their Properties . . . . . 352

11.8 Comparison of Solutions and Stochastic Population Control 363 
11.9 Caculation of Solutions to Population RSDE . . . . . . 372

12 Maximum Principle for Stochastic Systems with Jumps 377

12.1 Introduction . . . . . . . . . . . . . . . . 377

12.2 Basic Assumption and Notation . . . . . . . . . . . . . . 378

12.3 Maximum Principle and Adjoint Equation as BSDE with

Jumps . . . . . . . . . . . . . . . . . . . . . . . 379

12.4 A Simple Example . . . . . . . . . . . . . . . . . . 380

12.5 Intuitive thinking on the Maximum Principle . . . . . . . 381

12.6 Some Lemmas. . . . . . . . . . . . . . . . . . . . . 383

12.7 Proof of Theorem $354 \ldots \ldots \ldots \ldots$. . . . . . . . . 386

A A Short Review on Basic Probability Theory $\quad 389$

A.1 Probability Space, Random Variable and Mathematical Expectation . . . . . . . . . . . . . 389

A.2 Gaussian Vectors and Poisson Random Variables . . . . . . 392

A.3 Conditional Mathematical Expectation and its Properties . 395

A.4 Random Processes and the Kolmogorov Theorem . . . . . . 397

B Space D and Skorohod's Metric 401

C Monotone Class Theorems. Convergence of Random Processes407

C.1 Monotone Class Theorems . . . . . . . . . . . . . . . 407

C.2 Convergence of Random Variables ... . . . . . . . . 409

C.3 Convergence of Random Processes and Stochastic Integrals 411

$\begin{array}{ll}\text { References } & \mathbf{4 1 5}\end{array}$

$\begin{array}{ll}\text { Index } & \mathbf{4 3 1}\end{array}$ 


\section{Preface}

Stochastic differential equations (SDEs) were first initiated and developed by K. Ito (1942). Today they have become a very powerful tool applied to Mathematics, Physics, Chemistry, Biology, Medical science, and almost all sciences. Let us explain why we need SDEs, and how the contents in this book have been arranged.

In nature, physics, society, engineering and so on we always meet two kinds of functions with respect to time: one is determinstic, and another is random. For example, in a financial market we deposite money $\pi_{t}$ in a bank. This can be seen as our having bought some units $\eta_{t}^{0}$ of a bond, where the bond's price $P_{t}^{0}$ satisfies the following ordinary differential equation $d P_{t}^{0}=P_{t}^{0} r_{t} d t, P_{0}^{0}=1, t \in[0, T]$, where $r_{t}$ is the rate of the bond, and the money that we deposite in the bank is $\pi_{t}=\eta_{t}^{0} P_{t}^{0}=\eta_{t}^{0} \exp \left[\int_{0}^{t} r_{s} d s\right]$. Obviously, usually, $P_{t}^{0}=\exp \left[\int_{0}^{t} r_{s} d s\right]$ is non-random, since the rate $r_{t}$ is usually deterministic. However, if we want to buy some stocks from the market, each stock's price is random. For simplicity let us assume that in the financial market there is only one stock, and its price is $P_{t}^{1}$. Obviously, it will satisfy a differential equation as follows:

$$
d P_{t}^{1}=P_{t}^{1}\left(b_{t} d t+d(\text { a stochastic perturbation })\right), P_{0}^{1}=P_{0}^{1}, t \in[0, T],
$$
where all of the above processes are 1-dimensional. Here the stochastic perturbation is very important, because it influences the price of the stock, which will cause us to earn or lose money if we buy the stock. One important problem arises naturally. How can we model this stochastic perturbation? Can we make calculations to get the solution of the stock's price $P_{t}^{1}$, as we do in the case of the bond's price $P_{t}^{0}$ ? The answer is positive, usually a 
continuous stochastic perturbation will be modeled by a stochastic integral $\int_{0}^{t} \sigma_{s} d w_{s}$, where $w_{t}, t \geq 0$ is the so-called Brownian Motion process (BM), or the Wiener process. The 1-dimensional BM $w_{t}, t \geq 0$ has the following nice properties: 1) (Independent increment property). It has an independent increment property, that is, for any $0<t_{1}<\cdots<t_{n}$ the system $\left\{w_{0}, w_{t_{1}}-w_{0}, w_{t_{2}}-w_{t_{1}}, \cdots, w_{t_{n}}-w_{t_{n-1}},\right\}$ is an independent system. Or say, the increments, which happen in disjoint time intervals, occured independently. 2) (Normal distribution property). Each increment is Normally distributed. That is, for any $0 \leq s<t$ the increment $w_{t}-w_{s}$ on this time interval is a normal random variable with mean $m$, and variance $\sigma^{2}(t-s)$. We write this as $w_{t}-w_{s} \sim N\left(m, \sigma^{2}(t-s)\right)$. 3) (Stationary distribution property). The probability distribution of each increment only depends on the length of the time interval, and it does not depend on the starting point of the time interval. That is, the $m$ and $\sigma^{2}$ appearing in property 3 ) are constants. 4) (Continuous trajectory property). Its trajectory is continuous. That is $\mathrm{BM} w_{t}, t \geq 0$ is continuous in $t$.

Since the simplest or say, the most basic continuous stochastic perturbation, intuitively will have the above four properties, the modeling of the general continuous stochastic perturbation by a stochastic integral with respect to this basic BM $w_{t}, t \geq 0$ is quite natural. However, the 1 -dimensional $\mathrm{BM}$ also has some strange property: Even though it is continuous in $t$, it is nowhere differentiable in $t$. So we cannot define the stochastic integral $\int_{0}^{t} \sigma_{s}(\omega) d w_{s}(\omega)$ for each given $\omega$. That is why K. Ito (1942) invented a completely new way to define this stochastic integral.

Our first task in this book is to introduce the Ito stochastic integral and discuss its properties for later applications.

After we have understood the stochastic integral $\int_{0}^{t} \sigma_{s}(\omega) d w_{s}(\omega)$ we can study the following general stochastic differential equation (SDE):

$x_{t}=x_{0}+\int_{0}^{t} \widetilde{b}\left(s, x_{s}\right) d s+\int_{0}^{t} \widetilde{\sigma}\left(s, x_{s}\right) d w_{s}, t \geq 0$,

or equivalently, we write

$$
d x_{t}=\widetilde{b}\left(t, x_{t}\right) d t+\widetilde{\sigma}\left(t, x_{t}\right) d w_{t}, x_{0}=x_{0}, t \geq 0 .
$$

Returning to the stock's price equation, we naturally consider it as the following SDE:

$$
d P_{t}^{1}=P_{t}^{1}\left(b_{t} d t+\sigma_{t} d w_{t}\right), P_{0}^{1}=P_{0}^{1}, t \in[0, T] .
$$

Comparing this to the solution of $P_{t}^{0}$, one naturally asks could the solution of this SDE be $P_{t}^{1}=P_{0}^{1} \exp \left[\int_{0}^{t} b_{s} d s+\int_{0}^{t} \sigma_{s} d w_{s}\right]$ ? To check this guess, obviously if we can have a differential rule to perform differentiation on $P_{0}^{1} \exp x_{t}$, where $x_{t}=\int_{0}^{t} b_{s} d s+\int_{0}^{t} \sigma_{s} d w_{s}$, then we can make the check. Or more generally, if we have an $f(x) \in C^{2}(R)$ and $d x_{t}=b_{t} d t+\sigma_{t} d w_{t}$, can we have

$$
d f^{\prime}\left(x_{t}\right)=f^{\prime}\left(x_{t}\right) d x_{t}=f^{\prime}\left(x_{t}\right)\left(b_{t} d t+\sigma_{t} d w_{t}\right) ?
$$


If as in the deterministic case, this differential rule holds true, then we immediately see that $P_{t}^{1}=P_{0}^{1} \exp \left[\int_{0}^{t} b_{s} d s+\int_{0}^{t} \sigma_{s} d w_{s}\right]$ satisfies (2). Unforturnately, such a differential rule is not true. K. Ito (1942) has found that it should obey another differential rule - the so-called Ito's formula:

$d f^{\prime}\left(x_{t}\right)=f^{\prime}\left(x_{t}\right) d x_{t}+\frac{1}{2} f^{\prime \prime}\left(x_{t}\right)\left|\sigma_{t}\right|^{2} d t$.

By this rule one easily checks that

$$
P_{t}^{1}=P_{0}^{1} \exp \left[\int_{0}^{t} b_{s} d s+\int_{0}^{t} \sigma_{s} d w_{s}-\frac{1}{2} \int_{0}^{t}\left|\sigma_{s}\right|^{2} d s\right]
$$

is a solution of $(2)$, and $\widetilde{P}_{t}^{1}=P_{0}^{1} \exp \left[\int_{0}^{t} b_{s} d s+\int_{0}^{t} \sigma_{s} d w_{s}\right]$ actually satisfies another SDE:

$$
d \widetilde{P}_{t}^{1}=\widetilde{P}_{t}^{1}\left[\int_{0}^{t} b_{s} d s+\int_{0}^{t} \sigma_{s} d w_{s}+\frac{1}{2} \int_{0}^{t}\left|\sigma_{s}\right|^{2} d s\right], \widetilde{P}_{0}^{1}=P_{0}^{1}, \forall t \in[0, T]
$$

Our second task in this book is to establish the Ito formula and discuss its applications: solving SDE and solving other problems.

However, even if we have a powerful Ito formula (or say, Ito's differential rule) in our hand, we still need to discuss how to solve the general SDE, because usually, the form of the solution of SDE is not easy to guess. Moreover, for solutions of SDE, we actually meet a more complicated and hence also a more interesting case. Consider some physical quantity $x_{t}$ determined by dynamics. If this dynamics is deterministic, that is, it is not disturbed by any random noises, say such that $d x_{t}=\widetilde{b}\left(t, x_{t}\right) d t, x_{0}=x_{0}, t \geq$ 0 ; then solving this ODE we immediately get this quantity $x_{t}$. However, if the dynamics are disturbed by some continuous random noise, say such that $d x_{t}=\widetilde{b}\left(t, x_{t}\right) d t+\widetilde{\sigma}\left(t, x_{t}\right) d w_{t}, x_{0}=x_{0}, t \geq 0$, then for the amount $x_{t}$, or say, the solution of this SDE, two situations can arise. The first one is, if we think that the random noise - BM $w_{s}, s \leq t$, is an input, then after disturbing the dynamics we get an output $x_{t}$. This means that the solution $x_{t}$ is a functional of the given noise - BM $w_{s}, s \leq t$ for each $t$. We will call such a solution a strong solution. Another situation is that for a given noise we cannot immediately find the solution. However, we can find a random process $\widetilde{x}_{t}, t \geq 0$, and maybe another random noise that is also a BM $\widetilde{w}_{t}, t \geq 0$, such that $\left(\widetilde{x}_{t}, \widetilde{w}_{t}\right), t \geq 0$ satisfy the SDE $d \widetilde{x}_{t}=\widetilde{b}\left(t, \widetilde{x}_{t}\right) d t+\widetilde{\sigma}\left(t, \widetilde{x}_{t}\right) d \widetilde{w}_{t}, \widetilde{x}_{0}=x_{0}, t \geq 0$. In this case we will call $\left(\widetilde{x}_{t}, \widetilde{w}_{t}\right), t \geq 0$ or simply, $\widetilde{x}_{t}, t \geq 0$, a weak solution of the original SDE. Obviously, from the engineering point of view the strong solutions is more realistic and useful. However, since, if the strong solution $x_{t}, t \geq 0$ exists, then all finite dimensional probability ditributions of $\left(x_{t}, w_{t}\right)_{t>0}$ are the same as that of $\left(\widetilde{x}_{t}, \widetilde{w}_{t}\right)_{t \geq 0}$. So, if we do not know the existence of a strong solution, but we do know the existence of a weak solution $\left(\widetilde{x}_{t}, \widetilde{w}_{t}\right), t \geq 0$, then from the probability point of view the weak solution can still help us in some sense. Therefore, for solutions of SDEs there are two kinds that need to be considered: strong solutions and weak solutions.

Our third task in this book is to introduce the concepts of solutions and to discuss their existence and uniquess and the related important theory. (For example, Girsanov's theorem and the matingale representation theorem, the first of which can help us find weak solutions, while the second 
xiv

one is necessary for finding the solutions of backward SDE and the filtering problem considered later).

Since, actually, in the realistic world we will always meet some jump type stochastic perturbation, in this book we also consider stochastic integrals with respect to a Poisson counting measure (which is generated by a Poisson point process), the Ito formula and SDE for this case. (To find the reason why we consider the Poisson point process and its related integral as a jump type stochastic perturbation see the subsection "The General Model and its Explanation" in Chapter 8 - "Option Pricing in a Financial Market and BSDE").

The first three Chapters are intended to solve the above three tasks. They are the basic foudation of the SDE theory and its applications.

However, interesting and important things for SDE do not only come from the above mentioned three chapters, where they exhibit the following facts: the definition of Ito's stochastic integrals and Ito's differential rule are completely different from the deterministic case, etc. The interesting and important things also come from the following facts:

1) For an ordinary differential equation (ODE) $d x_{t}=\widetilde{b}\left(t, x_{t}\right) d t, x_{0}=$ $x_{0}, t \geq 0$ if $\widetilde{b}(t, x)$ is only bounded and jointly continuous, then even though the solution exists, is not necessary unique. However, for the SDE (1) in 1 -dimensional case if $\widetilde{b}(t, x)$ and $\widetilde{\sigma}(t, x)$ are only bounded and jointly Borelmeasurable, and $|\widetilde{\sigma}(t, x)|^{-1}$ is also bounded and $\widetilde{\sigma}(t, x)$ is Lipschitz continuous in $x$, then (1) will have a unique strong solution. (Here "strong" means that $x_{t}$ is $\mathfrak{F}_{t}^{w}$-measurable). This means that adding a non-degenerate stochastic perturbation term into the differential equation, can even improve the nice property of the solution.

2) The stochastic perturbation term has an importaqnt practical meaning in some cases and it cannot be discarded. For example, in the investment problem and the option pricing problem from a Financial Market, the investment portfolio actually appears as the coefficient of the stochastic integral in an SDE, where the stochastic integral acts like a stochatic perturbation term.

3) The solutions of SDEs and backward SDEs can help us to explain the solutions of some deterministic partial differential equations (PDEs) with integral terms (the Feynman-Kac formula) and even to guess and find the solution of a PDE, for example, the soluition of the PDE for the price of a option can be solved by a solution of a BSDE - the Black-Scholes formula.

4) More and more.

So we have many reasons to study the SDE theory and its applications more deeply and carefully. That is why we have a Chapter that discusses useful tools for SDE, and a Chapter for the solutions of an SDE with nonLipschitzian coefiicients. These are Chapter 4 and 5 .

The above concerns the first part of our book, which represents the theory and general background of the SDE. 
The second part of our book is about the Applications.

We first provide a short Chapter to help the reader to take a quick look at how to use Stochastic Analysis (the theory in the first part), to solve an SDE.

Then we discuss the estimation problem for a signal process : the so-called filtering problem, where the general linear and non-linear filtering problem for continous SDE systems and SDE systems with jumps, the KalmanBucy filtering equation for continuous systems, and the Zakai equation for non-linear filtering, etc. are also considered.

Since, now, research on mathematical finance, and in particular on the option pricing problem for the financial market has become very popular, we also provide a Chapter that discusses the option pricing problem and backward SDE, where the famous Black-Scholes formulas for a market with or without jumps are derived using a probability and a PDE approach, respectively; and the arbitrage-free market is also discussed. The interesting thing here is that we deal with the mathematical financial problem by using the backward stochastic differential equation (BSDE) technique, which now becomes very powerful when treating many problems in the finacial market, in mathematics and in other sciences.

Since deterministic population control has proved to be important and efficient, and the stochastic population control is more realistic, we also provide a Chapter that develops the stochastic population control problem by using the reflecting SDE approach, where the existence, the comparison and the calculation of the population solution and the optimal stochastic population control are established.

Besides, the stochastic Lagrange method for the stochastic optimal control, the non-linear pathwise stochastic optimal control, and the Maximum Principle (that is, the necessary conditions for a stochastic optimal control) are also formulated and developed in specific Chapters, respectively.

For the convenience of the readers three Appendixes are also provided: giving a short review on basic probability theory, space D and Skorohod's metric, and monotone class theorems and the convergence of random processes.

We suggest that the reader studies the book as follows:

For readers who are mainly interested in Applications, the following approach may be considered: Appendix $\mathrm{A} \rightarrow$ Chapter $1 \rightarrow$ Chapter $2 \rightarrow$ Chapter $3 \rightarrow$ Chapter $6 \rightarrow$ Any Chapter in The second part "Applications" except Chapter 10, and at any time return to read the related sections in Chapters 4 and 5, or Appendixes B and C, when necessary. However, to read Chapter 10, knowledge of Chapters 4 and 5 and Appendixes B and C are necessary. 


\section{Acknowledgement}

The author would like to express his sincere thanks to Professor Alan Jeffrey for kindly recommending publication of this book, and for his interest in the book from the very beginning to the very end, and for offering many valuable and important suggestions. Without his help this book would not have been possible 


\section{Abbreviations and Some Explanations}

All important statements and results, like, Definitions, Lemmas, Propositions, Theorems, Corollaries, Remarks and Examples are numbered in sequential order throughout the whole book. So, it is easy to find where they are located. For example, Lemma 22 follows Definition 21, and Theorem 394 is just after Remark 393, etc. However, the numbers of equations are arranged independently in each Chapter and each Appendix. For example, (3.25) means equation 25 in Chapter 3 , and (C.4) means the equation 4 in Appendix C.

The following abbreviations are frequently used in this book.

a.e. almost everywhere.

a.s. almost sure.

BM Brownian Motion.

BSDE backward stochastic differential equation.

FSDE forward stochastic differential equation.

H-J-B equation Hamilton-Jacobi-Bellman equation.

IDE integral-differential equation.

ODE ordinary differential equation.

PDE partial differential equation.

RCLL right continuous with left limit.

SDE stochastic differential equation.

$P-a . s$ almost sure in probability $P$.

$a^{+} \quad \max \{a, 0\}$.

$a^{-} \quad \max \{-a, 0\}$.

$a \vee b \quad \max \{a, b\}$.

$a \wedge b \quad \min \{a, b\}$. 
$\mu<<\nu$ measure $\mu$ is absolutely continuous with respect to $\nu$; that is, for any measurable set $A, \nu(A)=0$ implies that $\mu(A)=0$.

$\xi_{n} \rightarrow \xi$, a.s. $\xi_{n}$ converges to $\xi$, almost surely; that is, $\xi_{n}(\omega) \rightarrow \xi(\omega)$ for all $\omega$ except at the points $\omega \in \Lambda$, where $P(\Lambda)=0$.

$\xi_{n} \rightarrow \xi$, in $P \quad \xi_{n}$ converges to $\xi$ in probability; that is, $\forall \varepsilon>0$, $\lim _{n \rightarrow \infty} P\left(\omega:\left|\xi_{n}(\omega)-\xi(\omega)\right|>\varepsilon\right)=0$.

$\#\{\cdot\}$ the numbers of $\cdot$ counted in the set $\{\cdot\}$.

$\sigma\left(x_{s}, s \leq t\right)$ the smallest $\sigma$-field, which makes all $x_{s}, s \leq t$ measurable. $E[\xi \mid \eta] E[\xi \mid \sigma(\eta)]$. It means the conditional expectation of $\xi$ given $\sigma(\eta)$.

The following notations can be found on the corresponding pages. For example, $\mathfrak{F}, 4,387$ means that notation $\mathfrak{F}$ can be found in page 4 and page 387.

$\Omega, 4,387$

$\mathfrak{F}, 4,387$

$\mathfrak{F}_{t}, 4$

$\mathcal{F}_{p}, 34$

$\mathcal{F}_{p}^{1}, 34$

$\mathcal{F}_{p}^{2}, 34$

$\mathcal{F}_{p}^{2, l o c}, 34$

$F_{\mathcal{F}}^{2}\left(R^{d \otimes d_{2}}\right), 212$

$\mathcal{L}^{0}, 43$

$\mathcal{L}_{T}^{0}, 43$

$\mathcal{L}^{2}, 43$

$\mathcal{L}_{T}^{2}, 43$

$\mathcal{L}^{2, l o c}, 48$

$\mathcal{L}_{M}^{2}, 50$

$\mathcal{L}_{M}^{2, \text { loc }}, 51$

$L_{\mathfrak{F}}^{2}\left(R^{d \otimes d_{1}}\right), 212$

$L_{\pi(\cdot)}^{2}\left(R^{d \otimes d_{2}}\right), 212$

$L^{1}(\Omega, \mathfrak{F}, P) .12$

$\mathcal{M}^{2}, 35$

$\mathcal{M}^{2, c}, 37$

$\mathcal{M}^{2, l o c}, 35$

$\mathcal{M}^{2, \text { rcll }}, 37$

$\mathcal{M}_{T}^{2}, 35$

$\mathcal{M}_{T}^{2, c}, 37$

$\mathcal{M}_{T}^{2, l o c}, 35$

$\mathcal{M}_{T}^{2, \text { rcll }}, 37$

$\mathcal{O}, 7$

$\mathcal{P}, 7$

$P, 4,387$

$S_{\mathfrak{F}}^{2}\left(R^{d}\right), 212$ 


\section{Part I}

\section{Stochastic Differential \\ Equations with Jumps in \\ $R^{d}$}

